\title{
RICE CROP MAPPING USING SENTINEL-1A PHENOLOGICAL METRICS
}

\author{
C.F. Chen*, N.T. Son, C.R. Chen, L.Y. Chang, S.H. Chiang \\ Center for Space and Remote Sensing Research, National Central University, \\ Jhongli District, Taoyuan City 32001, TAIWAN \\ cfchen@csrsr.ncu.edu.tw, ntsonait@hotmail.com, ccruapes@gmail.com, lychang@csrsr.ncu.edu.tw, gilbert@csrsr.ncu.edu.tw
}

\section{Commission WG VIII/8}

KEY WORDS: Sentinel-1A, crop phenology, rice crop mapping, Mekong Delta.

\begin{abstract}
:
Rice is the most important food crop in Vietnam, providing food more than 90 million people and is considered as an essential source of income for majority of rural populations. Monitoring rice-growing areas is thus important to developing successful strategies for food security in the country. This paper aims to develop an approach for crop acreage estimation from multi-temporal Sentinel-1A data. We processed the data for two main cropping seasons (e.g., winter-spring, summer-autumn) in the Mekong River Delta (MRD), Vietnam through three main steps: (1) data pre-processing, (3) rice classification based on crop phenological metrics, and (4) accuracy assessment of the mapping results. The classification results compared with the ground reference data indicated the overall accuracy of $86.2 \%$ and Kappa coefficient of 0.72 . These results were reaffirmed by close correlation between the government's rice area statistics for such crops $\left(\mathrm{R}^{2}>0.95\right)$. The values of relative error in area obtained for the winter-spring and summer-autumn were $-3.6 \%$ and $6.7 \%$, respectively. This study demonstrates the potential application of multi-temporal Sentinel-1A data for rice crop mapping using information of crop phenology in the study region.
\end{abstract}

\section{INTRODUCTION}

Rice is the most important food crop in Vietnam, providing food more than 90 million people and is an essential source of income for majority of rural populations. More than $80 \%$ of the exported rice amount was from the Mekong River Delta (MRD), South Vietnam. Due to pressures of population growth, rice production in the study region has been intensified to meet people's food demands and economic development. Crop intensification not only brings benefits to farmers, but also triggers issues of environmental degradation and yield reduction due to soil depletion. Monitoring rice-growing areas is thus important to developing strategies for food security in the region.

Previous studies of rice crop monitoring in the region were carried using coarse resolution satellite data, including MODIS and ENVSIAT data. The use of MODIS reveals advantages due to its wide coverage and high temporal resolution. However, there are also limitations due to data contamination caused by cloud cover and mixed-pixel issues. This problem can be partly overcome by ENVISAT data. However, this satellite mission ended on 08 April 2012, following the unexpected loss of contact with the satellite. The Sentinel-1A satellite launched on 3 April 2014 gives the opportunity to collectively map small rice fields at different scales because the data have high spatial resolution of $10 \mathrm{~m}$ and temporal resolution of 12 days.

This paper aims to develop an approach for monitoring rice-growing areas from multi-temporal Sentinel-1A data. We processed the data for two main rice crops of winter-spring and summer-autumn seasons in 2015 in MRD, South Vietnam.

\section{STUDY AREA}

The study region (MRD) covers approximately 40,000 $\mathrm{km}^{2}$. Majority of the area was allocated for rice production. The climate is humid subtropical monsoon with an annual mean temperature of $27^{\circ} \mathrm{C}$. There are two distinct seasons in the region: dry season (December-April) and rainy season (MayNovember). The region is ideal for rice production due to its fertile alluvial soils, high solar radiation, favourable and stable temperature, and available water resource for irrigation around the year. There are five cropping seasons per year, but winterspring (November-December to February-March) and summerautumn (April-May to July-August) are the two main crops that produce a majority of rice production. The rice varieties with cultivation times of $90-110$ days were commonly cultivated in the study region.

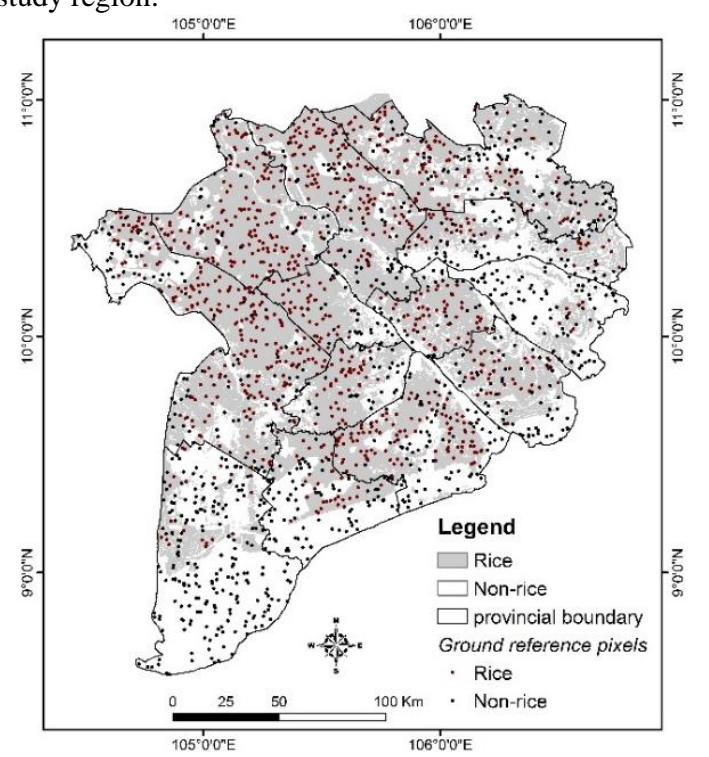

Figure 1. The location of the study region. Points extracted from the ground reference data were used for accuracy assessment.

\footnotetext{
* Corresponding author
} 


\section{DATA COLLECTION}

The Sentinel-1A data were acquired from the European Space Agency (ESA) for winter-spring and summer-autumn cropping seasons in 2015 from 30 Oct 2014 to 24 August 2015. The Sentinel-1A SAR instrument operates at $5.405 \mathrm{GHz}$ (C-band, corresponding to a radar wavelength of about $5.6 \mathrm{~cm}$ ), containing $\mathrm{VH}$ and VV polarizations with a revisit cycle of 12 days. We collected the 2006 MRD land-use/cover map from the GIS and Remote Sensing Research Center, Academy of Science and Technology, Vietnam and the 2012 map of rice cropping systems (Son et al., 2013).

The 2006 map was originally constructed using Landsat images and validated through ground survey results. These maps were updated for rice class and used to construct ground reference data used for accuracy assessment of the mapping results (Figure 2). The rice area statistics were also collected from General Statistics Office of Vietnam and used to verify the consistency with the mapping results.

\section{METHODS}

The pre-processing of Sentinel-1A data includes four main steps: (1) radiometric calibration to convert digital pixel values of VH/VV amplitude into sigma nought $\left(\sigma^{\circ}\right)$ values, (2) speckle noise filtering using Lee filter, (3) terrain correction, and (4) incidence angle normalization. This study used the time-series polarization ratio of $\mathrm{VH} / \mathrm{VV}$ for rice crop mapping in the study region.

To create the time-series VH/VV dataset for the period from 30 Oct 2014 to 24 August 2015, we first calculated the VH/VV ratio for every 12-day Sentinel-1 scene. These scenes were then stacked into a multi-temporal composite scene. The time-series $\mathrm{VH} / \mathrm{VV}$ data presenting speckle noise were filtered using the empirical mode decomposition (EMD) method (Huang et al., 1998).

This study used the normalized difference in values between sowing and heading dates index (NDSH) for rice crop mapping, calculated as follows:

$$
N D S H=\frac{H D-S D}{H D+S D},
$$

where, HD and SD are sowing and heading dates, respectively. In this study, we assumed the sowing period across the study region lasts approximately one month. These NDSH images were then stacked into one composite image.

To extract the rice growing areas, an image of maximum NDSH values was first calculated from the NDSH composite image. The receiver operating characteristic (ROC) curve (Metz, 1986; Zweig and Campbell, 1993) was then applied using ground reference data to obtain thresholds for mapping rice-growing areas in respect to two classes of rice and non-rice.

We assessed the mapping results using 1,000 pixels randomly extracted from ground reference data (Figure 1). The consistency of the mapping results was also verified with the rice areas statistics obtained from the government.

\section{RESULTS AND DISCUSSION}

The mapping results indicated the spatial distributions of rice growing areas for the winter-spring and summer-autumn seasons in the study region (Figure 2). In general, rice was cultivated in most of the study region, with more concentrated in the upper part of MRD. The spatial distributions of rice were relatively scattered along the coastal areas because rice in these two seasons were basically practiced in the dry season, and soil salinity intrusion was a limiting factor to rice production in areas along the coastal zone.

The classification maps were compared with the ground reference data using 1,000 pixels (randomly extracted from the ground reference data), indicating satisfactory results. The overall accuracy and Kappa coefficient were $86.2 \%$ and 0.72 , respectively. These results were reaffirmed by comparing rice areas obtained from Sentinel-1A data classification and the government's rice area statistics. The results indicated close consistency between the two datasets, in both growing seasons (Figure 3).

The relative error in area (REA) in percentage between these two datasets indicated a slightly underestimation, in both cases. The REA value obtained for the winter-spring crop was $3.6 \%$, while that for the summer-autumn crop was $-6.7 \%$. The larger error was observed for the summer-autumn crop probably attributed the effects of weather conditions due to the onset of wet season.
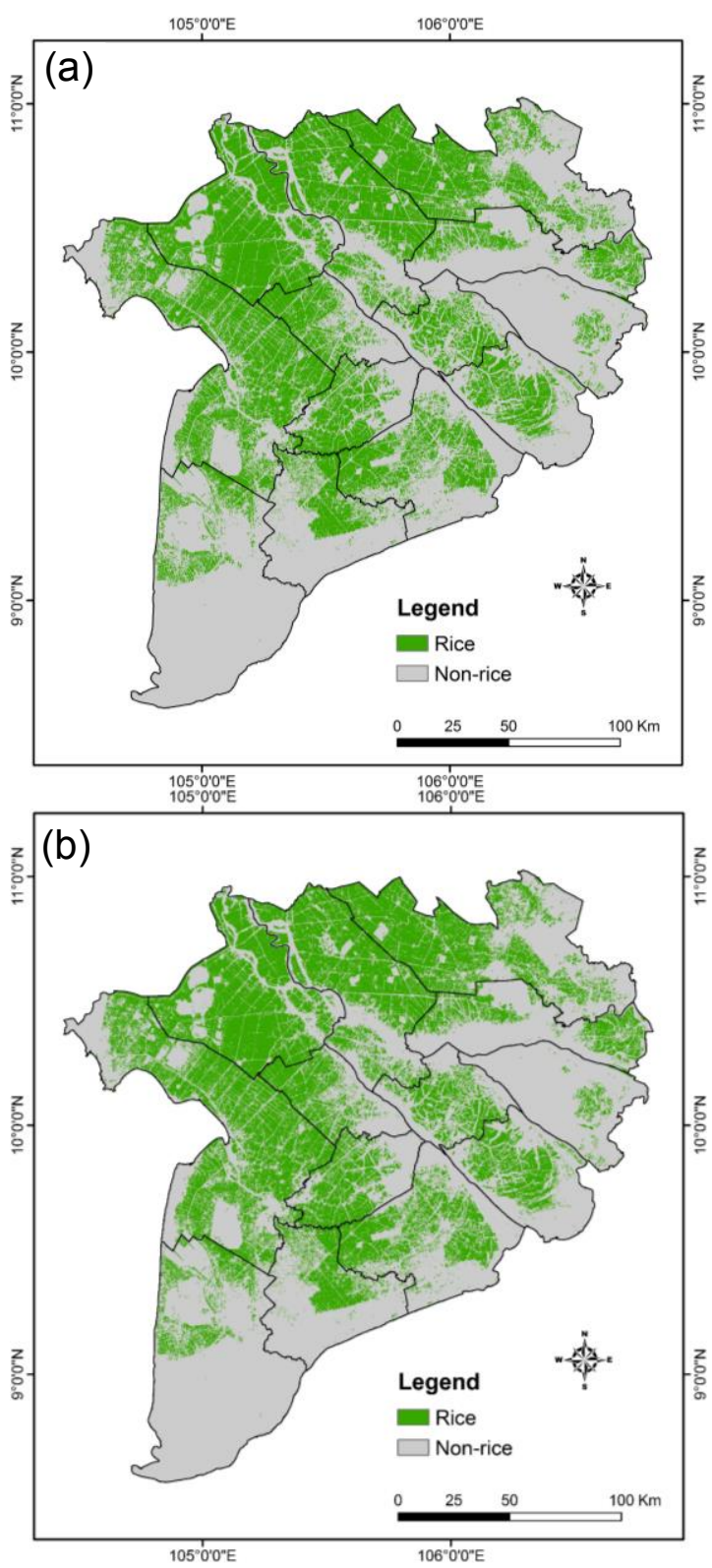

Figure 2. Spatial distributions of rice growing areas in 2015: (a) winter-spring crop, and (b) summer-autumn, autumn-winter crop. 

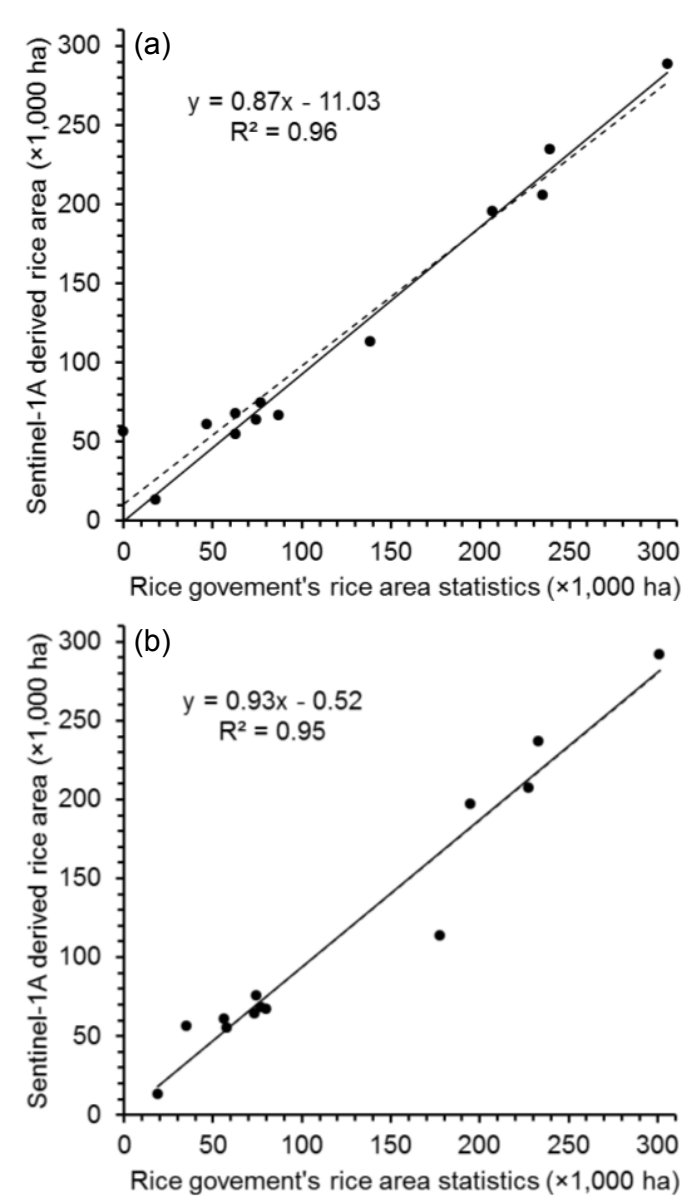

Figure 3. Correlation between the Sentinel-1A-derived rice area and the government's rice area statistics: (a) winter-spring crop, and (b) summer-autumn crop.

\section{CONCLUSION}

This study aimed to delineate rice-growing areas from multitemporal Sentinel-1A data in MRD, South Vietnam. The mapping results compared with the ground reference data confirmed the potential of our approach for rice crop mapping in the study region. The overall accuracy and Kappa coefficient values were $86.2 \%$ and 0.72 , respectively. The rice growing areas derived from the satellite data compared with the government's statistics reaffirmed the consistency between these two datasets. This study demonstrates the application of Sententinel-1A data for collectively mapping rice patches in the study region. The results could provide quantitative information on cropping areas for rice growing areas, which was useful for agronomic planners to monitor agricultural practices and production estimation in the region.

\section{REFERENCES}

Huang, N.E., Shen, Z., Long, S.R., Wu, M.C., Shih, H.H., Zheng, Q., Yen, N.C., Tung, C.C., Liu, H.H., 1998. The empirical mode decomposition and the Hilbert spectrum for nonlinear and non-stationary time series analysis. Proceedings of the Royal Society of London. Series A: Mathematical, Physical and Engineering Sciences 454, 903-995.

Metz, C.E., 1986. ROC methodology in radiologic imaging. Invest. Radiol. 21, 720-733.

Son, N.-T., Chen, C.-F., Chen, C.-R., Duc, H.-N., Chang, L.-Y., 2013. A Phenology-Based Classification of Time-Series
MODIS Data for Rice Crop Monitoring in Mekong Delta, Vietnam. Remote Sensing 6, 135-156.

Zweig, M.H., Campbell, G., 1993. Receiver-operating characteristic (ROC) plots: a fundamental evaluation tool in clinical medicine. Clin. Chem. 39, 561-577. 